\title{
Optimum Identification Method of Sorting Green Household Waste
}

\author{
Mohd Hisam Daud ${ }^{1, *}$ and Zol Bahri Razali ${ }^{1}$ \\ ${ }^{1}$ Robotics and Automation Technology, Faculty of Engineering Technology, Universiti \\ Malaysia Perlis, 02100 Padang Besar, Perlis, Malaysia
}

\begin{abstract}
This project is related to design of sorting facility for reducing, reusing, recycling green waste material, and in particular to invent an automatic system to distinguish household waste in order to separate them from the main waste stream. The project focuses on thorough analysis of the properties of green household waste. The method of identification is using capacitive sensor where the characteristic data taken on three different sensor drive frequency. Three types of material have been chosen as a medium of this research, to be separated using the selected method. Based on capacitance characteristics and its ability to penetrate green object, optimum identification method is expected to be recognized in this project. The output capacitance sensor is in analogue value. The results demonstrate that the information from the sensor is enough to recognize the materials that have been selected.
\end{abstract}

\section{Introduction}

The project aims to develop a material to be removed, in order to improve the concept od reduce, reuse and recycle. The aim is also to design a system to separate household waste objects to separate them from the main waste stream. In particular, it serves to distinguish some types of waste directly or automatically. A detector will be placed somewhere on the conveyor, the sensor is transmitted through the object to be distinguished, thresholding circuits or software to distinguish the objects located either above or below the level sensor which will decide what type of the detected object.

This research is to find the best identification method to sort co-mingled household waste material. Generally, the process of separating household waste of recyclable materials such as plastic, concrete, paper, forest green and others. However, there is some sort of technology is used, such as an infrared sensors and infrared laser to separate paper, concrete, plastic, and other. The techniques is used to analyze the waste materials based on size and shape of objects and in a recycling center facilities there is some of the techniques can be implemented. However, to meet current needs, vision systems or any other latest technique which can recognize different types of recycle material in household waste can be used as an alternative method. Optimum identification technique must be used to recognize the household waste material.

\footnotetext{
* Correcponding author : $\underline{\text { mhisam.daud@gmail.com }}$
} 


\section{Literature Review}

At present, Malaysia is running out of space to dispose of municipal solid waste generated each day from anthropogenic consumption. Based on a study by the Ministry of Housing and Local Government, the landfill in this country will be filled by $80 \%$ within a few years [1]. In fact, many serious enviromental problems that produce form landfills themselves that effect both for us and for future generations.

Malaysian daily average litter is $0.8 \mathrm{~kg}$ per person per day. Waste generated by Malaysia also noted the high rate among the countries of 15000 tons of garbage every day. If we collect all the waste and put in one place, we can fill with garbage KL twin towers in just 9.5 days. Even expected amount of waste will increase by $2 \%$ per year, depending on the activity of the population, economic activities and waste disposal methods [1].

The definition of 'waste' is taken from the Concise Oxford Dictionary refers to "useless remains" or value or lack of use is the product of human activities. Physically, useful products we can find the same ingredients but different in terms of the lack of useful production.

Various schemes could be classified for the waste: the physical state (liquid, solid, gas) and then in solid waste by the original use (food waste, packaging waste, etc.), materials (paper, glass, etc.) [2], with the physical characteristics (burning, composting, recycling), the origin (commercial, domestic, industrial, agricultural, etc.) or with the level of security (dangerous, not dangerous). Household and commercial waste is often referred to as Municipal Solid Waste (MSW) [3]. Household waste is one of the most difficult to manage the waste effectively. It consists of various types of materials (metal, paper, plastic, glass, organic) at all mixed together. MSW composition is also variable, both geographically and seasonally from city to rural areas and from country to country[2].

Green waste is biodegradable waste materials which may consist of a park or garden waste, such as flower or grass cuttings and hedge trimmings, as well as commercial and domestic food waste. The differentiation green identifying it as high in nitrogen, is contrary to brown waste, which is mainly carbon. Green waste is often collected through private waste management contractors business or municipal curbside collection schemes and subject to an independent audit. Biofuel can be produced from biodegradable green waste that captured from Biogas. non-food crops also can be used to produce cellulosic ethanol that from green waste [4].

Currently, optical sorters is used by the facility to sort the waste form green household waste, have what it takes to pre-shredded green waste streams, automatically sort and clean contaminated. The combination of mechanical pre-processing systems (air classification, size, grinding, etc.) are use in the technology to remove prohibitive contaminants (metals, plastics) from the green waste automatically, and generates a contaminant-free green waste product of composting or anaerobic digestion [5]. There are two main areas in green waste processing facility where optical sorters are in a position to provide added value to customers. The automated removal of any metals and plastics are two of the most common preferred application option. If there are needs to remove plastic contaminants from the food, there is a sorting facility features with a full spectrum near infrared (NIR) sensor to classify polymers and analyze and other organic materials at lower volumes [6]. This latest generation separator, with a pixel resolution of up to $16 \mathrm{x}$ optical sorters else, really provide optical isolation in high definition. 


\section{Methods and Materials}

\subsection{Process Flow Charts.}

Figure 1 shows a flow chart to describe the step to finish this project. The project starts with finding problems related to sorting household waste and what happens at present. Then study on the current project in sorting system, process, equipment such as sensors that. can detect material and any rules or principles involved in preparing the material that has been done. Review of the literature on the requirements and available methods or methods of concept will guide researchers to the idea of optimal design or the optimal method of preparing a variety of material systems. This process will continue by selecting materials from household waste as a medium in this study.

After selecting at least five types of waste as a medium of research, analysis, basic electrical properties of materials that have been done. Based on the results expected, an experimental study of the proposed methods will continue. After the results are compiled, comparing the identification method to choose the ingredients needed to make a difference in each method and then analyzing the optimum circuit identification method..

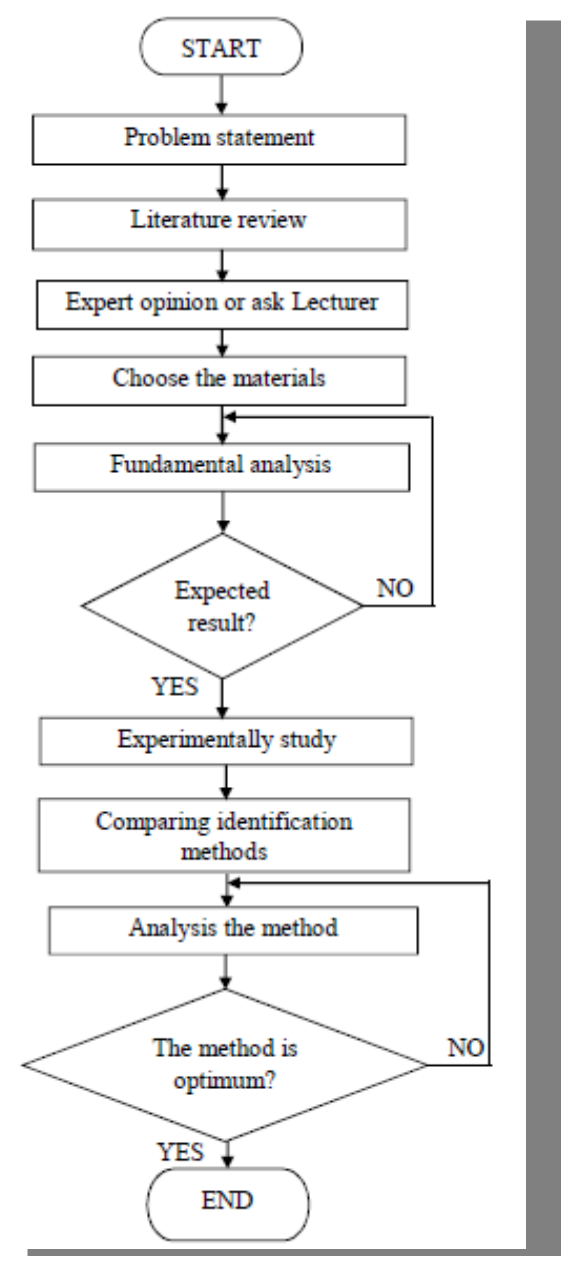

Fig.1. Flow Charts. 


\subsection{Analyze the Properties of Household Waste Materials.}

Outside, there is a lot of household waste, therefore for the purposes of this study, the authors only focused on the type of waste that can be separated into three types such as: metal, concrete and green forests. type of material has the properties of mechanical, chemical or electrical different [7]. Therefore, to run some analysis on materials properties is important, as it will cause some impact on the sensor used in this project.

\subsection{Selection of the Sorting Methods.}

The main part of the project is to find the best methods for distinguishing sorting waste materials are different. It refers to the best method that can detect and differentiate forest green, plastic, metal, concrete and paper at once. It can also be said that this technology will lead to a good choice for the development of the economic sustainability of recycling sorting facility [1].

Five methods were shortlisted. Basic system studied methods to determine its relationship with material properties [8]. It is important to understand their relationship because of the properties of the sensor to be used in the sorting system has the effect of material properties.

Based on data regarding the correlation of these traits, one of the best methods will be selected for further study on methods like, how it will be implemented, what event, what is the advantage compared to others. When a complete system is known, we will propose a redesign sorting system, with the system to be as optimal isolation system for the project, such as a system that can be compiled for different materials.

\subsection{Redesign the Sorting System.}

For this case, the focus is on the circuit that follows the order of the sorting process. For example, when the sensor detects the green woods, it returns a signal to the rest of the sorting bins and the waste products should be sorted into the correct bins.

\section{Results and Discussions}

\subsection{Analysis the Method of Sorting Using Capacitive Sensor.}

The data can be collected by measure the analog signals that detect from the capacitive sensor. The output of capacitive sensor must be in analog. When it detect present objects or the materials, the voltage value (analog signal) will show the different value depent on the capacitive objects. Microcontroller only could read the digital signal, so that the analog signal data must be convert to digital signal first and process the data. This process also known as analog to digital converter (ADC). Three type of frequency will be used in this analysis are $104.7 \mathrm{khz}, 96.6 \mathrm{khz}$ and $89.7 \mathrm{khz}$ to be tested within $20 \mathrm{~cm}$ of distance between the materials.

\subsection{Results of Different Materials.}

Table 1 and Figure 2 show the relationship between different materials at a frequency of $89.7 \mathrm{kHz}$ with different distances. proximity sensor with surface materials up to $20 \mathrm{~cm}$ wide. The value that is different from the production of metal, wood, and concrete can be seen at a distance of $6 \mathrm{~cm}$ to $20 \mathrm{~cm}$. 
Table 1. Different Materials at $89.7 \mathrm{kHz}$.

\begin{tabular}{|c|c|c|c|}
\cline { 2 - 4 } \multicolumn{1}{c|}{} & \multicolumn{3}{c|}{ Type of material } \\
\hline Distance (cm) & Concrete & Metal & Wood \\
\hline 1 & 0 & 0 & 0 \\
\hline 2 & 0 & 0 & 0 \\
\hline 3 & 0 & 0 & 0 \\
\hline 4 & 2 & 155 & 77 \\
\hline 5 & 231 & 272 & 238 \\
\hline 6 & 352 & 332 & 310 \\
\hline 7 & 398 & 363 & 338 \\
\hline 8 & 416 & 380 & 336 \\
\hline 9 & 429 & 388 & 366 \\
\hline 10 & 434 & 400 & 351 \\
\hline 11 & 443 & 406 & 361 \\
\hline 12 & 445 & 408 & 370 \\
\hline 13 & 447 & 407 & 357 \\
\hline 14 & 448 & 411 & 360 \\
\hline 15 & 446 & 410 & 368 \\
\hline 16 & 445 & 409 & 371 \\
\hline 17 & 446 & 408 & 364 \\
\hline 18 & 445 & 414 & 379 \\
\hline 19 & 444 & 405 & 372 \\
\hline 20 & 444 & 401 & 369 \\
\hline
\end{tabular}

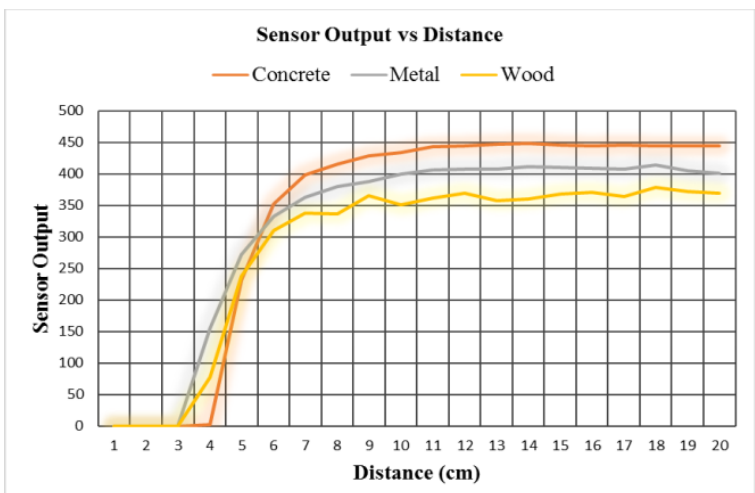

Fig. 2. Sensor output vs. Distance at $89.7 \mathrm{kHz}$.

Table 2 and Figure 3 shows the relationship between different materials at a frequency of $96.6 \mathrm{kHz}$ with a difference. Using the driving frequency of the sensor, the sensor output readings are almost equal to each other starting from a distance of $5 \mathrm{~cm}$ with a sensor surface of the material.

Table 3 and Figure 4 show the relationship between different materials on the frequency of $104.7 \mathrm{kHz}$ with a difference. Using the driving frequency of the sensor, the sensor output readings are almost equal to each other starting from $4 \mathrm{~cm}$ distance sensor with the material surface. 
Table 2. Different Materials at $96.6 \mathrm{kHz}$.

\begin{tabular}{|c|c|c|c|}
\cline { 2 - 4 } \multicolumn{1}{c|}{} & \multicolumn{3}{c|}{ Type of material } \\
\hline Distance (cm) & Concrete & Metal & Wood \\
\hline 1 & 0 & 0 & 0 \\
\hline 2 & 0 & 2 & 0 \\
\hline 3 & 216 & 648 & 49 \\
\hline 4 & 671 & 722 & 746 \\
\hline 5 & 738 & 746 & 745 \\
\hline 6 & 760 & 742 & 745 \\
\hline 7 & 764 & 749 & 749 \\
\hline 8 & 760 & 753 & 745 \\
\hline 9 & 758 & 748 & 746 \\
\hline 10 & 762 & 749 & 743 \\
\hline 11 & 760 & 750 & 747 \\
\hline 12 & 759 & 749 & 744 \\
\hline 13 & 756 & 750 & 743 \\
\hline 14 & 764 & 748 & 747 \\
\hline 15 & 762 & 754 & 744 \\
\hline 16 & 757 & 755 & 743 \\
\hline 17 & 758 & 753 & 740 \\
\hline 18 & 755 & 754 & 750 \\
\hline 19 & 762 & 753 & 747 \\
\hline 20 & 760 & 757 & 742 \\
\hline
\end{tabular}

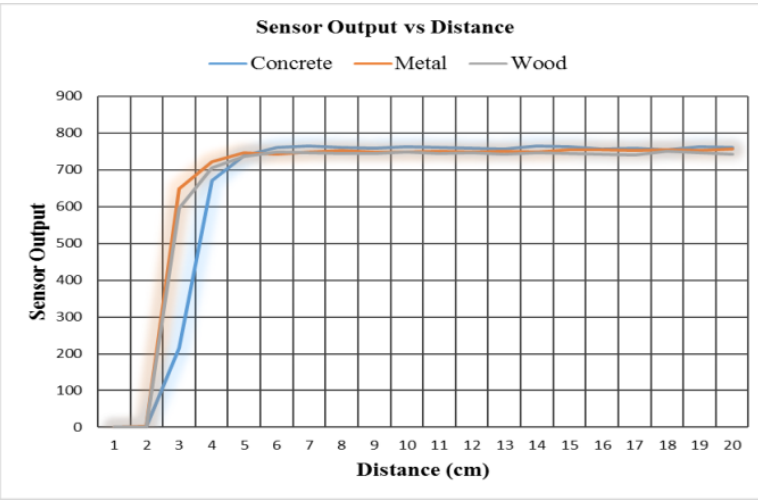

Fig. 3. Sensor output vs. Distance at $96.9 \mathrm{kHz}$.

\subsection{Comparing the methods.}

The comparison between all the methods is to determine which one is the optimum and the best method. Table 4 shows the different method that could recognize multi materials. This analysis come out with different result from different type method. Eddy current separator is only could sort the permanent magnet materials [9] and it can recognize ferrous and nonferrous materials (metallic material). Machine vision system can recognize the materials based on size, shape and color. The picture of materials will be taken to analyze it. Both capacitive proximity and capacitve sensor could detect all concrete, wood and metal but capacitive proximity sensor is not the best choice cause this sensor only detect the materials but could not recognize the materials because of the signal is digital value (' 0 ' and ' 1 '). The capacitve sensor is the best choice cause this sensor is the detect based on the analog value. 
Means different type of materials will show the different value of data. Therefore, the best method to sort and recognize the materials is capacitive sensor and it have potential to be the optimal method.

Table 3. Different Materials at $104.7 \mathrm{kHz}$.

\begin{tabular}{|c|c|c|c|}
\cline { 2 - 4 } \multicolumn{1}{c|}{} & \multicolumn{3}{c|}{ Type of material } \\
\hline Distance (cm) & Concrete & Metal & Wood \\
\hline 1 & 0 & 0 & 0 \\
\hline 2 & 0 & 686 & 373 \\
\hline 3 & 712 & 850 & 819 \\
\hline 4 & 859 & 878 & 871 \\
\hline 5 & 881 & 884 & 881 \\
\hline 6 & 885 & 889 & 881 \\
\hline 7 & 912 & 885 & 886 \\
\hline 8 & 893 & 887 & 882 \\
\hline 9 & 893 & 884 & 887 \\
\hline 10 & 887 & 885 & 881 \\
\hline 11 & 888 & 889 & 883 \\
\hline 12 & 885 & 886 & 878 \\
\hline 13 & 886 & 888 & 880 \\
\hline 14 & 911 & 884 & 883 \\
\hline 15 & 892 & 885 & 880 \\
\hline 16 & 892 & 882 & 886 \\
\hline 17 & 887 & 883 & 881 \\
\hline 18 & 887 & 888 & 883 \\
\hline 19 & 884 & 884 & 880 \\
\hline 20 & 885 & 887 & 881 \\
\hline
\end{tabular}

\section{Sensor Output vs Distance}

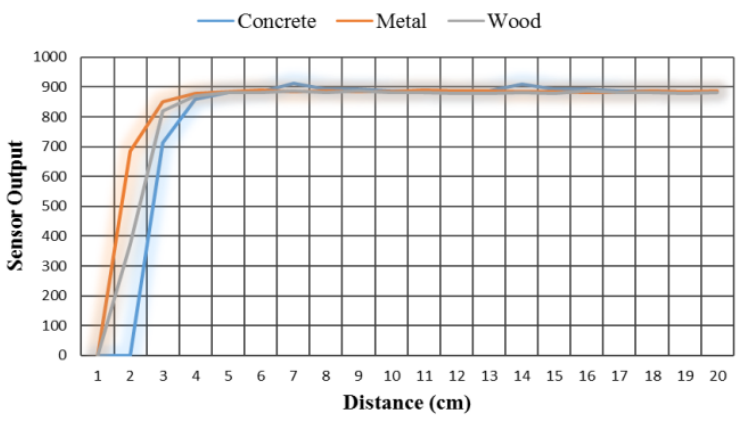

Fig. 4. Sensor output vs. Distance at $104.7 \mathrm{kHz}$.

Table 4. Recognizing various of materials.

\begin{tabular}{|c|c|c|c|}
\cline { 2 - 4 } \multicolumn{1}{c|}{} & \multicolumn{3}{c|}{ Materials } \\
\hline Method & Concrete & Metal & Green wood \\
\hline Eddy current separator & - & $\checkmark$ & - \\
\hline Capacitive proximity sensor & $\checkmark$ & $\checkmark$ & $\checkmark$ \\
\hline Machine vision & - & - & - \\
\hline Capacitive sensor & $\checkmark$ & $\checkmark$ & $\checkmark$ \\
\hline
\end{tabular}




\section{Discussions}

In general, sorting waste systems will sort thr recyclable waste resulting from household and in the same time it could solve the problem regarding to landfills that require a big space to dispose the garbage. Especially in urban areas that produce more waste compare to the village area or rural area. The sorting system required suitable method to make it applicable to all types of household waste or various type of waste. From this study, there are several methods available and can recognize the type, size, shape and color. More importantly, you want to use the methods that should be able to recognize and classify the type of waste in certain materials.

Image processing in machine vision methods are not suitable for use in sorting this material to identify various types of waste as image processing can only recognize by shapes, sizes, and colors. This system requires an application that can recognize the various types of waste to classify material following classes of materials.

Capacitive sensors are more suitable than other methods because the sensor can recognize the various types of materials based on the electrical properties of the material. Each material will produce different electrical property values. Capacitive sensors using eddy current principle that makes it react just like the concept of eddy currents. Eddy current principle also react when there is any material having electrical characteristics close to the investigation that resulted in the electromagnetic field.

\section{Conclusion}

This paper presented the method that can recognize three materials of household waste (concrete, metal and green woods). The capacitive sensor is the best identification method based on the characteristic of the sensor to sorting multi-material of household waste. This sensor can recognize three of materials using various frequencies. Based on the results, the frequency of $89.7 \mathrm{kHz}$ is the best to reach out to the capacitive sensor to analyze the types of waste materials to complete the rest of the household.

Data obtained using three household waste (concrete, metal and forest green). Preferred materials have different electrical properties in which the sensor can recognize and classify it. The large gap between the sensor output to concrete, metal and wood, green materials to make classification easier. If we use a capacitive proximity sensor, the sensor can detect each of the materials, however, because of different distances on the surface of the material; digital signal must give an inaccurate value.

Finally, the capacitive sensor is the most effective and efficient method to compare with others that can be used to separate the various materials as the method can recognize a variety of materials.

This work was financially supported by the Universiti Malaysia Perlis Seed Money (9014-00034).

\section{References}

1. Z.B. Razali, N.A. Mat Nawi, Automated Segregating Device for Reuse and Recycle Waste (Lambert, Chicago, 2013)

2. Z.B. Razali, G.B. Lee, High-End Technology for Effective Glass Manufacturing Industry (Lambert, Chicago, 2013)

3. P.C. Nicholas, Handbook of Solid Waste Management and Waste Minimization Technologies (Butterworth-Heinemann, Boston, 2003)

4. http://www.magsep.com/optical-sorting-applications/municipal-solid-waste-mswsorting/green-waste-sorting/ Retrieved on 24 November 2013. 
5. N. Kirchner, D. Hordern, D. Liu, G. Dissanayake, Sensors and Actuators A. Phys., 148, $96(2008)$

6. M.O. Rahman, A. Hussain, E. Scavino, H. Basri, M.A. Hannan, Expert Syst. with Appl., 38, 10398 (2011)

7. D. Fletcher, R. Gerber, IEEE Trans. Magn. 29, 3255 (1993)

8. L. Zitouni, R. Beheim, Huez, F. Belloir, IEEE Sens. J., 6, 1580 (2006)

9. M. Brojboiu, L. Mandache, V. Ivanov, 8th International Symposium on Advanced Topics in Electrical Engineering (2013) 